Journal of

Applied

Crystallography

ISSN 0021-8898

Editor: Anke R. Kaysser-Pyzalla

\title{
Design and application of a microfluidic device for protein crystallization using an evaporation-based crystallization technique
}

\section{Yong Yu, Xuan Wang, Dominik Oberthür, Arne Meyer, Markus Perbandt, Li Duan and Qi Kang}

J. Appl. Cryst. (2012). 45, 53-60

Copyright (C) International Union of Crystallography

Author(s) of this paper may load this reprint on their own web site or institutional repository provided that this cover page is retained. Republication of this article or its storage in electronic databases other than as specified above is not permitted without prior permission in writing from the IUCr.

For further information see http://journals.iucr.org/services/authorrights.html

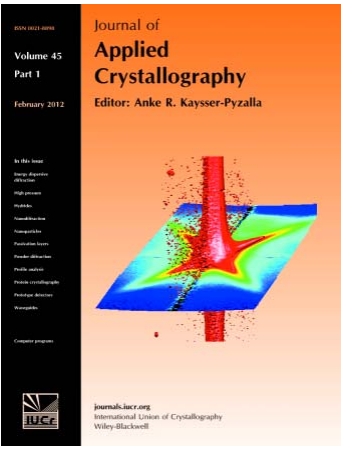

Journal of Applied Crystallography covers a wide range of crystallographic topics from the viewpoints of both techniques and theory. The journal presents papers on the application of crystallographic techniques and on the related apparatus and computer software. For many years, the Journal of Applied Crystallography has been the main vehicle for the publication of small-angle scattering papers and powder diffraction techniques. The journal is the primary place where crystallographic computer program information is published.

Crystallography Journals Online is available from journals.iucr.org 
Journal of

Applied

Crystallography

ISSN 0021-8898

Received 7 July 2011

Accepted 11 November 2011

(C) 2012 International Union of Crystallography

Printed in Singapore - all rights reserved

\section{Design and application of a microfluidic device for protein crystallization using an evaporation-based crystallization technique}

\author{
Yong Yu, ${ }^{\text {a* }}$ Xuan Wang, ${ }^{a}$ Dominik Oberthür, ${ }^{\mathrm{b}}$ Arne Meyer, ${ }^{\mathrm{c}}$ Markus Perbandt, ${ }^{\mathrm{b}}$ Li \\ Duan $^{\mathrm{a}}$ and Qi Kang ${ }^{\mathrm{a}}$
}

\begin{abstract}
${ }^{a}$ Key Laboratory of Microgravity (National Microgravity Laboratory), Institute of Mechanics, Chinese Academy of Sciences, Beijing 100190, People's Republic of China, $\mathbf{b}$ Institute of Biochemistry and Molecular Biology, University of Hamburg, c/o DESY, 22603 Hamburg, Germany, and 'Laboratory for Structural Biology of Infection and Inflammation, University of Lübeck, c/o DESY, 22607 Hamburg, Germany. Correspondence e-mail: yuyong@imech.ac.cn
\end{abstract}

\begin{abstract}
A new crystallization system is described, which makes it possible to use an evaporation-based microfluidic crystallization technique for protein crystallization. The gas and water permeability of the used polydimethylsiloxane (PDMS) material enables evaporation of the protein solution in the microfluidic device. The rates of evaporation are controlled by the relative humidity conditions, which are adjusted in a precise and stable way by using saturated solutions of different reagents. The protein crystals could nucleate and grow under different relative humidity conditions. Using this method, crystal growth could be improved so that approximately $1 \mathrm{~mm}$-sized lysozyme crystals were obtained more successfully than using standard methods. The largest lysozyme crystal obtained reached $1.57 \mathrm{~mm}$ in size. The disadvantage of the good gas permeability in PDMS microfluidic devices becomes an advantage for protein crystallization. The radius distributions of aggregrates in the solutions inside the described microfluidic devices were derived from in situ dynamic light scattering measurements. The experiments showed that the environment inside of the microfluidic device is more stable than that of conventional crystallization techniques. However, the morphological results showed that the protein crystals grown in the microfluidic device could lose their morphological stability. Air bubbles in microfluidic devices play an important role in the evaporation progress. A model was constructed to analyze the relationship of the rates of evaporation and the growth of air bubbles to the relative humidity.
\end{abstract}

\section{Introduction}

Despite many investigations, there is no accurate theory to substitute for empirical approaches in protein crystallization. Space was thought to be a perfect environment for the production of high-quality protein crystals because sedimentation movement and convective flow due to gravity are negligible under microgravity conditions (Yu et al., 2010; McPherson, 1999). However, experimentation in space has restricted access and high costs. In addition, a gravity-like disturbance (' $g$ jitter') also appears in spacecrafts. In fact, to create true microgravity conditions is difficult. Although experiments carried out in space have produced good results, Earth-based methods always have obvious attractions (Helliwell \& Chayen, 2007). The magnetic field (Sazaki, 1997; Yin et al., 2004, 2008) and gel methods (García-Ruiz et al., 2001) have been used to reduce the effect of convective flow on protein crystallization. Microfluidics also can provide the advantages of microgravity on Earth.
With the significant development of MEMS (microelectromechanical system) technology, microfluidics is currently one of the most rapidly growing frontier fields. Microfluidics offers unequaled experimental conditions to explore the complexity of protein crystallization (Leng \& Salmon, 2009). Using this technique the transport phenomena can be effectively controlled since there are no buoyancy-driven convection instabilities at such small length scales (Leng \& Salmon, 2009). This technique can also minimize the consumption of a protein sample and reduce the labor involved (Zheng, Tice \& Ismagilov, 2004; Hansen et al., 2002). The uncommon conditions involved, which include the reduction of the volumes of the crystallizers, growth in confined geometries, mononuclear nucleation etc., bring a new understanding of the nucleation and growth mechanisms during protein crystallization (Leng \& Salmon, 2009).

The first microfluidic device for high-throughput screening of protein crystallization conditions was designed using the free-interface diffusion method (Hansen et al., 2002, 2006). 
Ismagilov and co-workers also proposed droplet-based microfluidics to screen crystallization conditions of proteins, which exploits the possibility of generating aqueous nanolitresized droplets carried in an inert oil stream (Zheng et al., 2003, 2005; Zheng, Tice, Roach \& Ismagilov, 2004; Yadav et al., 2005). The use of a microdialysis-based microfluidic device was another important technique for protein crystallization (Shim, Cristobal, Link, Thorsen \& Fraden, 2007; Shim, Cristobal, Link, Thorsen, Jia et al., 2007), which used dialysis to supersaturate the protein solution. The protein crystals grown in polydimethylsiloxane (PDMS) (Hansen et al., 2006) and polymethylmethacrylate (Sauter et al., 2007) devices can be analyzed directly on-chip by X-ray diffraction.

At present, microfluidic devices can be used for screening and optimizing the protein crystallization conditions and to perform high-throughput data acquisition. Under these conditions, crystallization trials are implemented using microfluidic devices or conventional microbatch or vapor diffusion approaches (Hansen et al., 2002). However, appropriate microfluidic devices that can substitute for the conventional crystallization methods are still few in number.

Evaporation-based crystallization techniques are by far the most important of these methods. The droplet-based microfluidic device (Zheng, Tice, Roach \& Ismagilov, 2004) used the vapor diffusion method to screen and optimize the protein crystallization conditions. Talreja et al. (2005) manufactured an evaporation-based crystallization platform for protein crystallization, in which the rate of evaporation is controlled by diffusion through a capillary channel, but fabrication of this microfluidic device is complex.

This paper proposes a new microfluidic device for protein crystallization, consisting of a circular cavity made by a PDMS-glass system; the height is $100 \mu \mathrm{m}$. It can reduce the buoyancy-driven convection to control the growth of crystals. The diameter of the cavity in the horizontal plane reaches $10 \mathrm{~mm}$, which ensures that enough molecules are available to allow the growth of protein crystals. PDMS is the core material of many microfluidic chips and is permeable to gas and water. This characteristic was thought to be a major disadvantage in previously developed PDMS microsystems dedicated to protein crystallization (Lounaci et al., 2006). Lounaci et al. (2007) had to use water to control the relative humidity to about $100 \%$ around PDMS microsystems. In the present paper, the gas permeability of PDMS was used to evaporate the protein solution in the microfluidic device. The rates of evaporation were controlled by the relative humidity conditions, which were adjusted in a precise and stable way by using saturated solutions of different reagents. Using this microfluidic device, the protein crystals' growth could be controlled and improved by applying an evaporation-based crystallization technique. The radius distributions of aggregates in the solutions inside the described microfluidic devices were derived from in situ dynamic light scattering measurements. Morphological research into protein crystals grown in the microfluidic devices was also carried out to study the instability of the protein crystallization. The rates of evaporation influence the growth of crystals. The air bubbles in the microfluidic devices play an important role in the evaporation progress. A model was constructed to analyze the relationship of the rates of evaporation and the growth of air bubbles to the relative humidity.

\section{Experimental}

\subsection{The microfluidic devices}

The diffusion Grashof number $\mathrm{Gr}_{\mathrm{D}}$, a dimensionless number, is used to describe the buoyancy convection produced in the process of crystal growth. Its physical meaning is the ratio of buoyancy to viscous force in inhomogeneous media. The smaller the Grashof number, the smaller the buoyancy convection. The formula is

$$
\mathrm{Gr}_{\mathrm{D}}=\left(g L^{3} / v^{2}\right) \beta_{\mathrm{C}} \Delta C,
$$

where $g$ is the acceleration due to gravity, $v$ is the kinematic viscosity, $\beta_{\mathrm{C}}$ is the solution expansivity coefficient and $\Delta C$ is the concentration difference. $L$ is the characteristic length; the dimensions or height of the solution in the gravity direction can be used as the value of $L$. The diffusion Grashof number can be changed by changing one or more of three parameters: $g, v$ and $L$.

The production of protein crystals in space is a direct method that restrains buoyancy convection. The method whereby gels are added into a solution to increase the kinematic viscosity $v$ can also reduce the convective flow (GarcíaRuiz et al., 2001). Decreasing the characteristic length $L$ is another effective method.

For example, if the height of the solution in which the protein crystals grow is $2 \mathrm{~mm}$, then the characteristic length $L=2 \mathrm{~mm}$. The diffusion Grashof number on Earth is

$$
\mathrm{Gr}_{\mathrm{D}}^{\mathrm{E}}=\left(g L^{3} / v^{2}\right) \beta_{\mathrm{C}} \Delta C .
$$

The gravity level in an experimental satellite is about $1 \times$ $10^{-4} \mathrm{~g}$. Then the diffusion Grashof number in the space microgravity environment is

$$
\mathrm{Gr}_{\mathrm{D}}^{\mathrm{mg}}=10^{-4}\left(g L^{3} / v^{2}\right) \beta_{\mathrm{C}} \Delta C=10^{-4} \mathrm{Gr}_{\mathrm{D}}^{\mathrm{E}} .
$$

If the diffusion Grashof number was decreased to the level of the space microgravity environment by using a microchannel, the height of the microchannel $L_{\mathrm{m}}$ should be given by

$$
10^{-4}\left(g L^{3} / v^{2}\right) \beta_{\mathrm{C}} \Delta C=\left(g L_{\mathrm{m}}{ }^{3} / v^{2}\right) \beta_{\mathrm{C}} \Delta C,
$$

i.e. $L_{\mathrm{m}}=93 \mu \mathrm{m}$.

This shows that a microchannel can effectively restrain buoyancy convection. According to the viewpoint of fluid mechanics, the interior of the microfluidic device could be thought of as a microgravity environment. At low buoyancy, there is no difference between this kind of device and other microfluidic devices.

The microfluidic devices used in this work were designed as shown in Fig. 1. The depth of the microfluidic devices is $100 \mu \mathrm{m}$. The width of the microchannels is $1 \mathrm{~mm}$ and the diameter of the crystallization area is $10 \mathrm{~mm}$. The microfluidic devices were fabricated by multilayer soft lithography techniques using PDMS. A mixture of liquid prepolymer PDMS 
Table 1

The concentration of lysozyme crystal solutions.

\begin{tabular}{lll}
\hline & Lysozyme $\left(\mathrm{mg} \mathrm{ml}^{-1}\right)$ & $\mathrm{NaCl}\left(\mathrm{mol} \mathrm{l}^{-1}\right)$ \\
\hline 1 & 28 & 0.78 \\
2 & 19.25 & 0.75 \\
3 & 19.25 & 0.6 \\
4 & 16.75 & 0.495 \\
\hline
\end{tabular}

and its cross link agent at a proportion of about 10:1 was poured onto a mold and cured at $353 \mathrm{~K}$ for two hours. The solidified PDMS structures were then peeled off, and small holes were drilled into the PDMS layers using a borer to produce inlets and outlets. The PDMS pieces, laid on top of glass slides, were subsequently exposed to oxygen plasma for about $4 \mathrm{~min}$, so that the PDMS and glass layers were impacted together. Finally, the ensemble was heated again to $353 \mathrm{~K}$ for half an hour, which led to the formation of an irreversible PDMS-glass system.

The PDMS-PDMS systems were made by first assembling components consisting of an undrilled PDMS film (i.e. a film without microchannels) and a glass slide. Then the PDMS pieces containing microchannels and the PDMS-glass components without microchannels were assembled in a manner similar to that described above, such that all of the inside faces of the microchannel are PDMS material.

The thickness of the PDMS layers can influence the rate of evaporation. If the PDMS layers are thinner, the center of the crystallized area becomes dented easily and the insertion of tubes into the inlets and outlets becomes difficult. If the PDMS layers are much thicker, peeling off the solidified PDMS layers becomes difficult. The thickness of the PDMS layers was therefore controlled to be about 3.35 (7) $\mathrm{mm}$ and that of the PDMS films was about 1.18 (10) $\mathrm{mm}$.

\subsection{Reagents and methods}

An HAc-NaAc buffer solution (250 $\left.\mathrm{mmol} \mathrm{l}^{-1} \mathrm{pH} 4.5\right)$ was used, and chicken egg-white lysozyme was purchased from
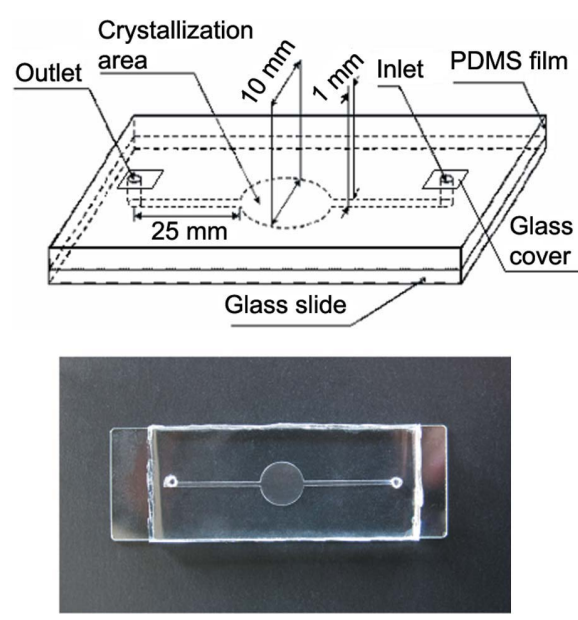

Figure 1

Schematic (top) and actual image (bottom) of the microfluidic device.
Table 2

The relative humidity of some saturated reagent solutions at $298 \mathrm{~K}$.

\begin{tabular}{lllll}
\hline Reagents & $\left(\mathrm{NH}_{4}\right)_{2} \mathrm{SO}_{4}$ & $\mathrm{KI}$ & $\mathrm{Mg}\left(\mathrm{NO}_{3}\right)_{2} \cdot 6 \mathrm{H}_{2} \mathrm{O}$ & $\mathrm{MgCl}_{2} \cdot 6 \mathrm{H}_{2} \mathrm{O}$ \\
\hline Relative humidity (\%) & $89(5)$ & $75(5)$ & $58(5)$ & $41(5)$
\end{tabular}

Sigma. High-purity deionized water (18.2 M $\Omega$ ) was obtained by passing distilled water through a Milli-Q Plus water purification system, and all other reagents were of analytical grade.

The buffer solution was filtrated with a $0.22 \mu \mathrm{m}$ filtration membrane before performing the experiments, and appropriate amounts of lysozyme powder and $\mathrm{NaCl}$ were then dissolved separately in buffer solution. The initial protein and $\mathrm{NaCl}$ concentrations used in the experiments are shown in Table 1. These solutions were kept at $277 \mathrm{~K}$. The lysozyme solution was centrifuged at $14000 \mathrm{r} \mathrm{min}^{-1}$ for about $15 \mathrm{~min}$, and then only the upper clear part of the solution was used in the experiments.

The $\mathrm{NaCl}$ solution and clear lysozyme solution were mixed together well. Then the mixture was injected into the microfluidic devices. The inlets and outlets were sealed using vaseline oil and then covered with cover glasses.

The protein solution easily produces and retains air bubbles, and small air bubbles enter easily into the microfluidic devices. Because the volume of the microchannel is small, the temperature of the solution inside of the microfluidic device is easily influenced by the ambient temperature. During this part of the experiments, up to the point where the PDMS devices are placed in a biochemical incubator (see below), the ambient temperature and that of the solutions should be higher than the controlled temperature inside of the biochemical incubator to avoid producing unwanted nucleation.

As shown in Fig. 2, the fabricated microfluidic devices were subsequently enclosed in a glass dryer box sealed with vaseline. Using various saturated reagent solutions, the humidity in this box can be controlled precisely and kept stable throughout the experiment. The saturated reagent solutions used in this paper are shown in Table 2.

Before the microfluidic devices were placed in this box, the saturated reagent solutions were filled into the underside vessel of the box. The quantity of solution must be sufficient to ensure the stability of the relative humidity for the duration of the experiment, and better results are obtained if the deposition protrudes out of the water. A hygrometer was used to check the relative humidity. In particular, hygrometry varies with temperature, so it was necessary to keep the system at a stable temperature. To ensure these conditions were maintained, the box was kept in a biochemical incubator.

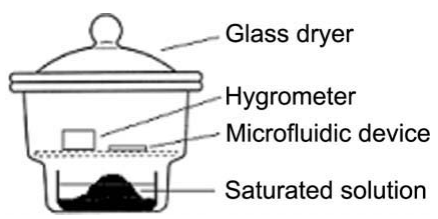

Figure 2

Schematic of the box for protein crystallization under controlled humidity conditions. 


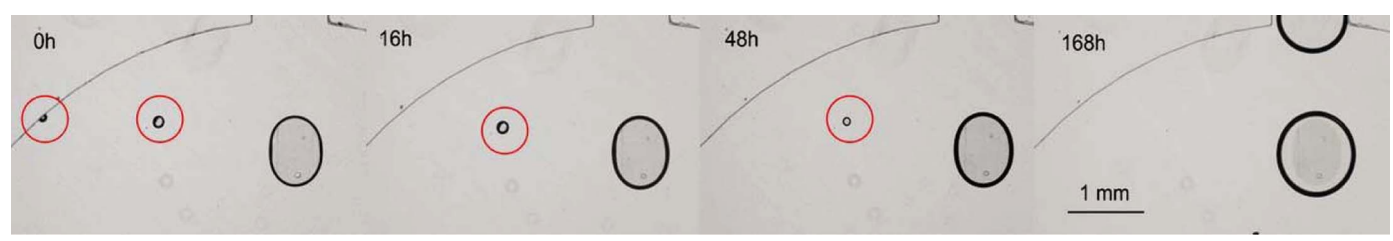

Figure 3

The growth of air bubbles in the microfluidic device at 89 (5)\% relative humidity. The growth conditions correspond to No. 4 in Table 1 , at 298.0 (5) K.

Once the values of relative humidity were stable, the box was removed from the incubator and a PDMS device was placed in the upper part of the box. Then the box was closed and put back into the biochemical incubator at 298.0 (5) K. Because PDMS is permeable to gas and water, the solution in the microfluidic devices can evaporate through the PDMS membrane to supersaturate the protein solution. Then nucleation can occur and protein crystals can grow. The rates of evaporation were controlled by controlling the relative humidity inside of the box. Observations were carried out using a microscope.

Simultaneously with the humidity-controlled experiments, dynamic light scattering (DLS) measurements on an identical microfluidic device were carried out in situ (Dierks et al., 2008) using a SpectroLight 500 (NABITEC GmbH, Germany). After the protein-salt solution was injected into the microfluidic device, the PDMS surfaces of the device were covered with a glass slide to seal it. It was then placed into the DLS instrumentation, in order to determine the aggregate sizes in the solution in the first four hours of protein crystal growth.

\section{Results and discussion}

\subsection{The evaporation of solution in the microfluidic devices}

Protein crystals grew after evaporation of the solution in the microfluidic devices. The rates of evaporation influence the crystal growth. These rates were controlled by the thickness of the PDMS layers and the different relative humidity conditions inside of the box. The higher the relative humidity, the lower the evaporation rates of the solution in the microfluidic devices. When the relative humidity was $89(5) \%$, the rates of the evaporation of the solution in the devices were very slow: it took about $528 \mathrm{~h}$ to dry all the areas of the devices. The growth of air bubbles in the microfluidic devices was influenced by the size of the bubbles under this relative humidity condition. Larger air bubbles would expand but smaller air bubbles would gradually contract (as shown in Fig. 3). When the relative humidity was $75(5) \%$, the rates of solution evaporation became slightly faster: it took about $264 \mathrm{~h}$ before all parts of the microfluidic device became dry. When the relative humidity was $58(5) \%$, the solution evaporation in all microfluidic devices exceeded $50 \%$ of the volume after about $192 \mathrm{~h}$. When the relative humidity was 41 (5)\%, air bubbles in the microfluidic devices became larger faster and all microfluidic devices were dry after cultivating for about $120 \mathrm{~h}$. The evaporation rates of the solution in the PDMS-PDMS microfluidic devices were a little quicker than those for the
PDMS-glass system because the PDMS films could be permeated by water vapor.

The results in Fig. 3 show that the air bubbles in the microfluidic devices play an important role in the evaporation process. A model was constructed to analyze the relationship of the rates of evaporation and the growth of air bubbles to the relative humidity. In this model it was supposed that the exchange of gas/solution occurred on the interface of the bubble with no involvement of PDMS. The water in solution and the vapor in the bubble exchanged with each other at the interface of the bubble. Then the vapor inside of the bubble exchanged with air outside of the microfluidic device via the PDMS membrane. This process is analyzed in detail in Appendix $A$. The analytical results can reasonably explain the phenomenon shown in Fig. 3. This model also implies that the larger the air bubbles, the larger the surface areas of evaporation and thus the faster the rates of evaporation. Though the evaporation rates mainly depend on the relative humidity conditions, they are also influenced by the sizes of the air bubbles in the microfluidic devices.

\subsection{Protein crystallization}

The lysozyme crystals shown in Fig. 4. were produced using the growth conditions in Table 1 and the relative humidities in

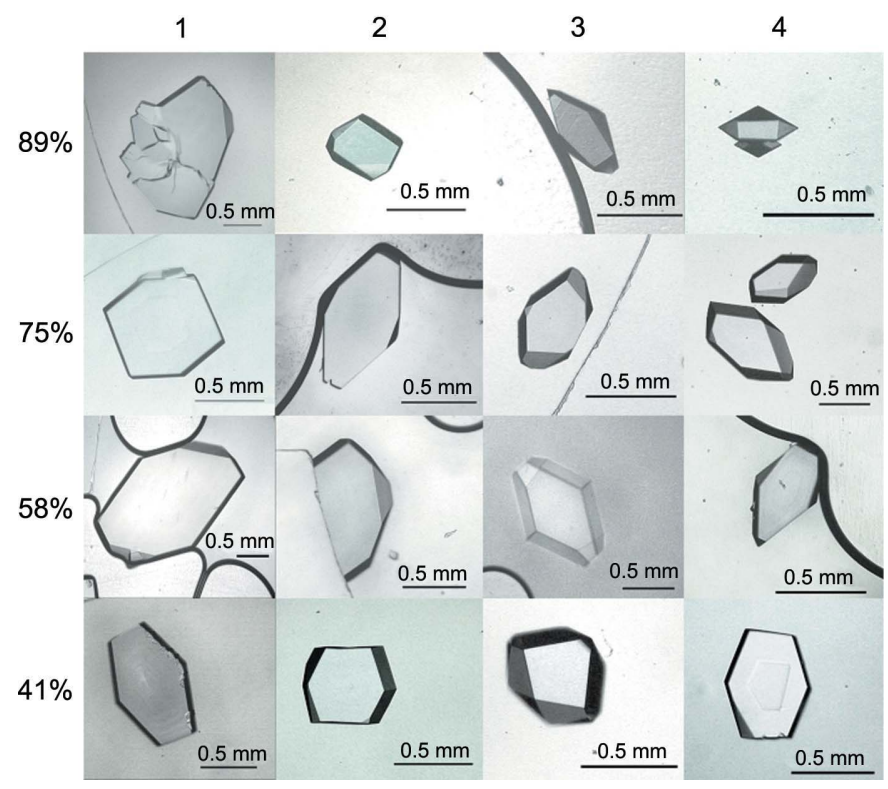

Figure 4

The lysozyme crystals obtained using a microfluidic device at 298.0 (5) K. The labels 1, 2, 3 and 4 correspond to the growth conditions in Table 1. 
Table 2. The experimental results showed that, under condition No. 1 and $41(5) \%$ relative humidity, the sizes of the lysozyme crystals reached around $1 \mathrm{~mm}$ and larger crystals were more easily obtained. Fig. 5(a) shows the largest lysozyme crystal obtained, which reached $1.57 \mathrm{~mm}$ in size. Fig. 6 demonstrates a case in which five lysozyme crystals, whose sizes were around $1 \mathrm{~mm}$, were obtained in one microfluidic device.

Using this technique one could study the instability mechanism of protein crystallization very well. The predominant means of mass transport inside of the microfluidic device is diffusion. When the mass transport is slower than the surface kinetics, and the transport process reaches the ratelimiting step, the protein crystal loses its morphological stability, which is detrimental to crystal quality. The occurrence of such instability also depends on crystal size and supersaturation (Heijna et al., 2007). The instability phenomenon cannot occur before the size of the lysozyme crystals exceeds a critical size. Nanev \& Penkova (2002) reported that the critical size of lysozyme crystals is $2.4-4.9 \mathrm{~cm}$ and that such sizes could not be reached experimentally, but in our experiments, the instability phenomenon could be observed after the size of lysozyme crystals reached several hundred micrometres. Since larger crystals were more easily obtained by using the microfluidic device described here, this technique

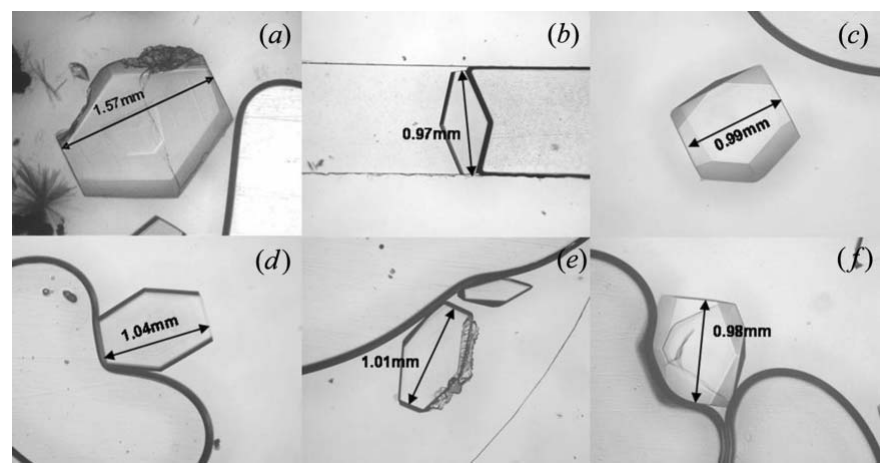

Figure 5

The lysozyme crystals obtained using a PDMS-glass microfluidic device at 298.0 (5) K. The growth conditions correspond to No. 1 in Table 1; the humidity was $41(5) \%$.

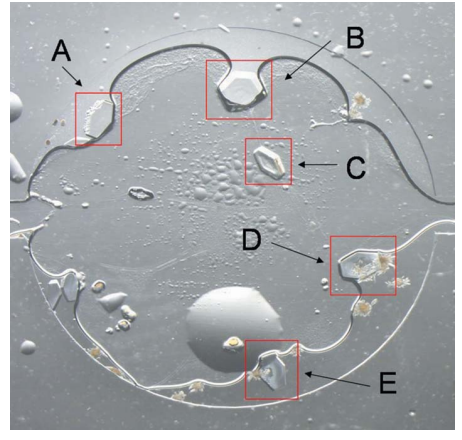

Figure 6

The lysozyme crystals in the PDMS-glass microfluidic device. The growth conditions correspond to No. 1 in Table 1; the humidity was $41(5) \%$. Sizes of the lysozyme crystals: A: $1.09 \mathrm{~mm}$; B: $1.15 \mathrm{~mm}$; C: $0.95 \mathrm{~mm}$; D: $1.13 \mathrm{~mm}$; E: $1.05 \mathrm{~mm}$.

has obvious advantages for studying the instability mechanism of protein crystallization. Fig. 7 shows images of lysozyme crystals that became morphologically unstable. Figs. 7(a) and $7(b)$ show that the lysozyme crystals have concave and reentrant corners appearing in the middle of the side faces because of a competition between two-dimensional nucleation at the edges of the crystal and step flow on its surface. Fig. 7(c) shows a lysozyme crystal that has some lattice defects, and in Fig. $7(f)$ the lysozyme crystal appears to have a radial shape. The crystals in Figs. 7(d) and 7(e) were obtained in the PDMSPDMS microfluidic device. There are sharp-angled salients appearing in the middle of the side faces. The growthpromoting center in the central part of the face means that the crystal growth should follow a spiral dislocation mechanism. According to the instability mechanism of inorganic crystals, it is not thought that this kind of instability could occur (Kuroda et al., 1977). This phenomenon is strange and the reasons for it are not clear. Could it be that vaporization of solution via every face affects the growth of lysozyme crystals? It may be caused by a particular instability mechanism that belongs to protein crystallization.

Larger crystals easily cleave or break into pieces, but using this kind of microfluidic device, the large crystals were not damaged when the microfluidic devices were moved. Because the microfluidic devices were enwrapping the protein crystals, the result was that the crystal obtained a hard shell.

However, if the evaporation process continues until all areas of the microfluidic devices are dry, the crystals will be damaged. Immersion of the microfluidic devices in paraffin oil can end the evaporation process and reserve a little solution to conserve the protein crystals, so after crystals have reached an optimal size, oils are added. The entire microfluidic device

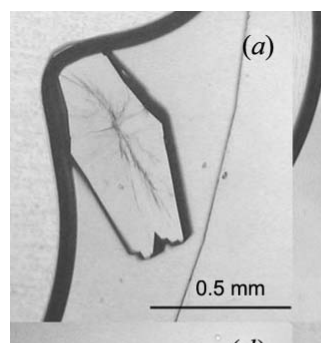

(d)

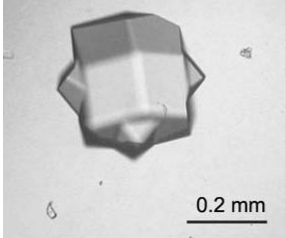

Figure 7

Images of different lysozyme crystals that became morphologically unstable, obtained at $298.0(5) \mathrm{K}$. The relative humidity of $(a),(b),(d)$ and $(e)$ was $58(5) \%$; the relative humidity of $(c)$ was $41(5) \%$ and the relative humidity of $(f)$ was $89(5) \%$. The growth conditions of $(a)$ and $(e)$ correspond to No. 1 in Table 1; the growth conditions of $(f)$ correspond to No. 2 in Table 1; the growth conditions of $(b),(c)$ and $(d)$ correspond to No. 4 in Table 1. $(d)$ and $(e)$ were grown using the PDMS-PDMS microfluidic device, while the other crystals were grown using the PDMSglass microfluidic device. 

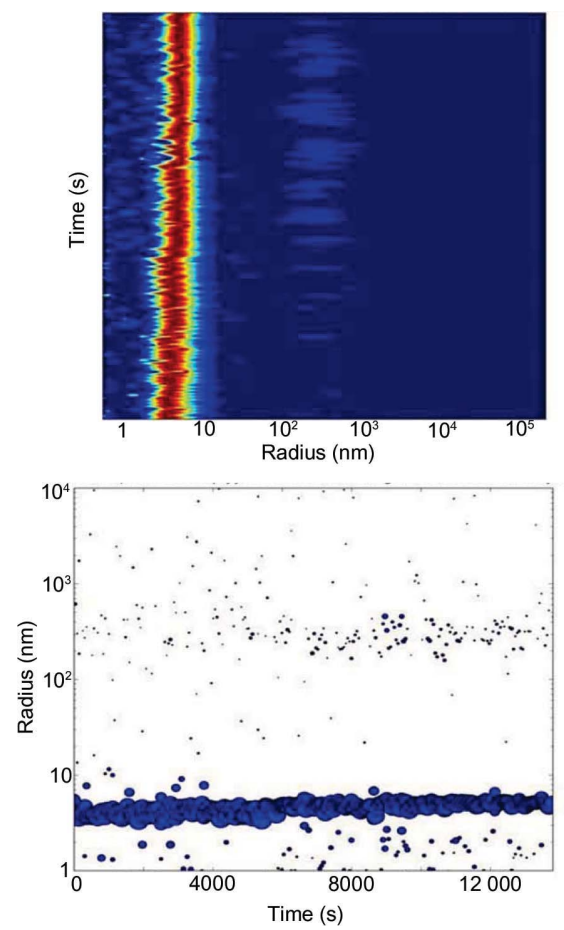
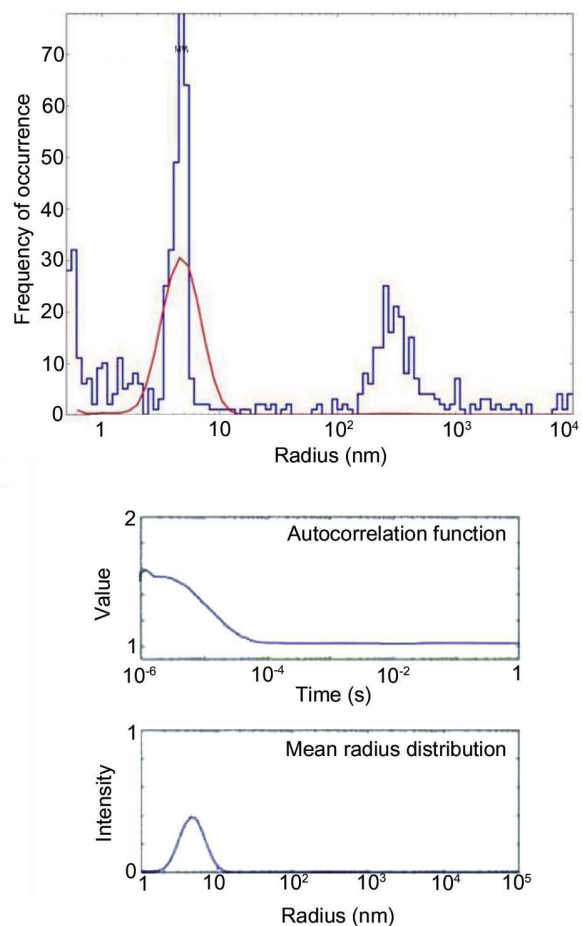

Figure 8

The display radius of the DLS measurement in the PDMS-glass microfluidic device. The growth conditions correspond to No. 1 in Table 1, 293.0 (5) K.

should be immersed in the paraffin oil. Experiments showed that the protein crystals in the microfluidic devices were not damaged after they had been immersed in paraffin oil for about 14 days.

\subsection{DLS measurement}

Fig. 8 shows that the sizes of most aggregates determined are less than $10 \mathrm{~nm}$. It is only towards the end of the experiment that large aggregates appeared. Diffusion is the dominate means of mass transport in the microfluidic devices. The diffusion rates of the large aggregates are slow. On the other hand, the depth of the microfluidic device is $100 \mu \mathrm{m}$, and the large aggregates are easily adsorbed on the wall of the device. Therefore, the environment inside the microfluidic device is clearer and more stable than that of conventional crystallization methods.

\section{Conclusions}

We have described here a crystallization system that makes it possible to combine microfluidics with evaporation-based techniques for protein crystallization. The gas and water permeability of the PDMS material was used and the relative humidity controlled by using saturated solutions of different reagents. This method allows nucleation and crystal growth under different relative humidity conditions. The believed disadvantage of the good gas permeability in PDMS material becomes an advantage. Using this method, crystal growth could be improved to the extent that approximately $1 \mathrm{~mm}$ sized lysozyme crystals were obtained. The largest lysozyme crystal acquired reached $1.57 \mathrm{~mm}$ in size. Though the size of protein crystals is not the most important factor in generating high-quality crystals, the growth of large lysozyme crystals is an important step. Large crystal size is often an indicator that you have a good crystal. Of course, the new technique should be assessed by $\mathrm{X}$-ray diffraction. In the future, the crystals grown within this microfluidic device should be analyzed directly onchip using X-rays.

The dynamic light scattering experiments showed that the environment inside of the microfluidic device is more stable and clearer than that of conventional crystallization methods. However, the morphological results showed that the protein crystals grown in the microfluidic device could lose their morphological stability, which is detrimental to crystal quality.

Because the microfluidic devices enwrap the protein crystals closer than conventional methods, giving the crystal a hard shell, these large crystals are not damaged by motion or transportation. This technique could be used to study the mechanism of protein crystallization in a microgravity environment, and as a substitute for protein crystallization experiments carried out in space. Though it uses a sample that is somewhat limited, the costs of using this technique are much less than that of producing protein crystals in space.

\section{APPENDIX $A$ \\ Analysis of the relationship of the rate of evaporation and the growth of air bubbles to the relative humidity}

Suppose the exchange of gas/solution occurred on the interface of the bubble with no involvement of PDMS, so the water in solution and the vapor in the bubble exchanged places with each other at the interface of the bubble. Then the vapor inside of the bubble is exchanged with air outside of the microfluidic device via the PDMS membrane.

First the relative humidity and the pressure inside of the box are discussed. Suppose the primary air in the box is pure dry air, the relative humidity inside the box is $0 \%$ and the pressure outside of the box is $P_{0}$. After the saturated reagent solutions have been added to the box, the relative humidity arrives at a balance. The relative humidity determined by the hygrometer is $B_{\mathrm{e}}$. The balanced pressure inside of the box is $P_{\mathrm{e}}$, and the volume of the box is $V_{\mathrm{e}}$. When the box is airtight and the balance is achieved, the relative humidity determined by a hygrometer equals the humidity of the saturated reagent solutions. The mass of the primary dry air $N_{0}$ in the box is

$$
N_{0}=P_{0} V_{\mathrm{e}} / R T
$$


for

$$
B_{\mathrm{e}}=N_{\mathrm{w}} /\left(N_{\mathrm{w}}+N_{0}\right)
$$

where $N_{\mathrm{w}}$ is the mass of vapor inside of the box, $R$ is the gas constant and $T$ is the temperature. After the balance is achieved, the total mass of air $N$ is

$$
N=\frac{N_{0}}{1-B_{\mathrm{e}}}=\frac{P_{0} V_{\mathrm{e}}}{\left(1-B_{\mathrm{e}}\right) R T}
$$

Under isothermal conditions,

$$
P_{\mathrm{e}} V_{\mathrm{e}}=N R T
$$

so

$$
\frac{P_{\mathrm{e}}}{P_{0}}=\frac{1}{1-B_{\mathrm{e}}} .
$$

This means that the pressure inside of the box is several times higher than atmospheric pressure under the high relative humidity conditions, which requires the box to be sealed very well. In practice, air could seep out of the box and the pressure inside the box could change. The released mass equals the evaporated mass of solution. Since the evaporation of the solution is a slow process, it is thought that the pressure inside of the box equals the atmospheric pressure and the relative humidity determined by a hygrometer equals the standard relative humidity of the saturated reagent solutions.

Fig. 9 shows the pressure balance of the bubble. For a surface tension $\sigma$, on the surface of a bubble whose radius is, we have

$$
P_{\mathrm{b}}=P_{\mathrm{t}}+P_{\mathrm{L}}=2 \sigma / R_{\mathrm{b}}+P_{\mathrm{L}} \text {. }
$$

Since the PDMS material is permeable to gas and the evaporation of the solution is slow, it is simply thought that the air in the bubble and the air in the box are connected. So

$$
P_{\mathrm{b}}=P_{\mathrm{e}}, \quad B_{\mathrm{b}}=B_{\mathrm{e}} \text {. }
$$

When the radius of the bubble is $50 \mu \mathrm{m}$ and the surface tension of water at $293 \mathrm{~K}$ is $0.073 \mathrm{~N} \mathrm{~m}^{-1}$, the added pressure as a result of the surface tension is

$$
P_{\mathrm{t}}=\frac{2 \sigma}{R_{\mathrm{b}}}=\frac{2 \times 0.073}{50 \times 10^{-6}}=2.93 \times 10^{3} \mathrm{~Pa} .
$$

Since $P_{\mathrm{t}}<<P_{0}$, the pressure balance of the bubble is achieved by the water pressure. The size of the bubbles is only controlled by the volume of the solution inside of the microfluidic device. The air pressure inside of the bubble is basically the same. If the air in the bubbles is superfluous, it diffuses into

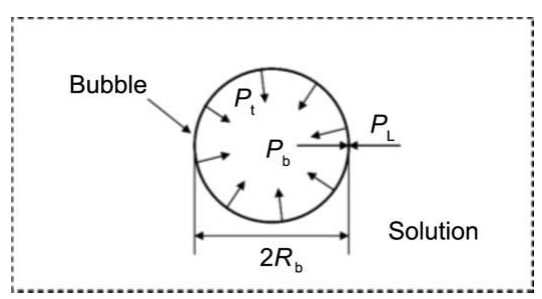

Figure 9

A schematic diagram of the pressure balance of the bubble. the box via the PDMS membrane. When the bubbles lack air, the air also enters the bubbles via the PDMS membrane.

There is a chemical potential $(\mu)$ balance between the solution $(\mathrm{L})$ and vapor $(\mathrm{g})$ that has a saturated vapor pressure. The solutions in the microfluidic device are dilute, and could be approximately regarded as water. We have

$$
\mu_{\mathrm{L}}=100 \mu_{\mathrm{g}} \text {. }
$$

When the evaporation occurs on the interface of the bubbles, the change of Gibbs free energy that one molecule experiences during the phase transition is

$$
\begin{aligned}
\Delta G & =\mu_{\mathrm{g}}\left(B_{\mathrm{e}}\right)-\mu_{\mathrm{L}} \\
& =k_{\mathrm{B}} T \ln \left(B_{\mathrm{e}}\right)+k \Delta H+\sigma \Delta A_{\mathrm{b}}-k_{\mathrm{B}} T \ln (100 \%) \\
& =k_{\mathrm{B}} T \ln \left(B_{\mathrm{e}}\right)+k \Delta H+\sigma \Delta A_{\mathrm{b}},
\end{aligned}
$$

where $k_{\mathrm{B}}$ is the Boltzmann constant, $\Delta H$ is the evaporation enthalpy of one water molecule and $\Delta A_{\mathrm{b}}$ is the change in the surface area of the air-water interface that is brought about by evaporation of one water molecule. It is known that the vaporization heat of one water molecule is higher than its free energy. Therefore, $\Delta G$ is positive if the molecule does not exchange energy with its surroundings during its phase transition, which indicates that the evaporation will not happen. In reality, the evaporation always results in absorption of heat, causing the decrease of the temperature of the evaporation molecule. During this phase transition, most of the energy is reinjected by the collision of the molecule with other surrounding molecules. Only a little energy is reinjected by the molecular free energy that drives the evaporation. The coefficient $k$ gives the ratio of reinjected energy into the system.

We can take one of the dome-shaped bubbles shown in Fig. 10 as an example to analyze the relationship between $\Delta A_{\mathrm{b}}$ and radius $R_{\mathrm{b}}$. The wettability of the material was ignored, and the contact angle was supposed to be $90^{\circ}$. The bulk and surface area of the bubble are, respectively, given by

$$
\begin{gathered}
V_{\mathrm{b}}=(2 / 3) \pi R_{\mathrm{b}}^{3}, \\
A_{\mathrm{b}}=2 \pi R_{\mathrm{b}}^{2} .
\end{gathered}
$$

We also have

$$
\begin{gathered}
R_{\mathrm{b}}=\left(A_{\mathrm{b}} / 2 \pi\right)^{1 / 2}, \\
V_{\mathrm{b}}=(2 / 3) \pi\left(A_{\mathrm{b}} / 2 \pi\right)^{3 / 2} .
\end{gathered}
$$

Differentiating with respect to time, we have

$$
\frac{\mathrm{d} V_{\mathrm{b}}}{\mathrm{d} t}=\frac{1}{2}\left(\frac{A_{\mathrm{b}}}{2 \pi}\right)^{1 / 2} \frac{\mathrm{d} A_{\mathrm{b}}}{\mathrm{d} t}=\frac{R_{\mathrm{b}}}{2} \frac{\mathrm{d} A_{\mathrm{b}}}{\mathrm{d} t} .
$$

Figure 10

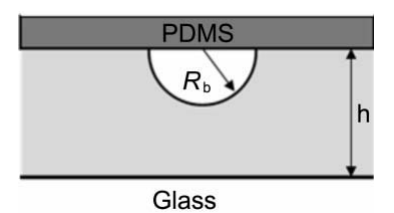

A dome-shaped bubble. 
Also

$$
\Delta V_{\mathrm{b}}=\frac{M_{\mathrm{w}} \Delta N}{\rho_{\mathrm{w}} N_{\mathrm{A}}},
$$

where $M_{\mathrm{w}}$ is the molar mass of water, $\rho_{\mathrm{w}}$ is the density of water, $N_{\mathrm{A}}$ is Avogadro's number and $\Delta N$ is the amount of gasification of water molecules. Differentiating with respect to time,

$$
\frac{\mathrm{d} V_{\mathrm{b}}}{\mathrm{d} t}=\frac{M_{\mathrm{w}}}{\rho_{\mathrm{w}} N_{\mathrm{A}}} \frac{\mathrm{d} N}{\mathrm{~d} t},
$$

so

$$
\begin{aligned}
\frac{R_{\mathrm{b}}}{2} \frac{\mathrm{d} A_{\mathrm{b}}}{\mathrm{d} t}=\frac{M_{\mathrm{w}}}{\rho_{\mathrm{w}} N_{\mathrm{A}}} \frac{\mathrm{d} N}{\mathrm{~d} t}, \\
\frac{\mathrm{d} A_{\mathrm{b}}}{\mathrm{d} N}=\frac{2 M_{\mathrm{w}}}{\rho_{\mathrm{w}} N_{\mathrm{A}}} \frac{1}{R_{\mathrm{b}}} .
\end{aligned}
$$

This means that, when $R_{\mathrm{b}}$ is smaller, $\Delta A_{\mathrm{b}}$ is larger, so it needs more interface energy.

Because $B_{\mathrm{e}}<1, \ln B_{\mathrm{e}}$ is negative. $\Delta H$ is constant. When the radius of a bubble $R_{\mathrm{b}}$ is small, $\sigma \Delta A_{\mathrm{b}}$ is larger and $\Delta G>0$. The result is that the vapor inside of the bubble forms as liquid and the bulk volume of the bubbles is contractible. When the radius of the bubble $R_{\mathrm{b}}$ is larger, $\sigma \Delta A_{\mathrm{b}}$ is small and $\Delta G<0$. The result is that the solutions inside of the microfluidic device vaporize and the bulk volume of bubbles increases.

According to the Arrhenius formula, the reaction rate constant for evaporation of water molecules is given by

$$
K=A \exp \left(\frac{-\Delta G}{k_{\mathrm{B}} T}\right),
$$

where $A$ is a constant. When $\Delta G<0$, the smaller $B_{\mathrm{e}}$ becomes, the larger $K$ becomes. The increasing rate of bubble growth equals the evaporation rate of the solution. So the increasing rate of bubble growth is

$$
\frac{\mathrm{d} V_{\mathrm{b}}}{\mathrm{d} t} \propto K S,
$$

where $V_{\mathrm{b}}$ is the bulk volume of bubbles and $S$ is the surface area of the air-water interface. This means that the lower the relative humidity is, the faster the increase of bubble mass becomes.

This work was supported by the National Natural Science Foundation of China (grant Nos. 10972224 and 11032011) and the Knowledge Innovation Program of the Chinese Academy of Sciences (grant No. KJCX2-YW-L08).

\section{References}

Dierks, K., Meyer, A., Einspahr, H. \& Betzel, C. (2008). Cryst. Growth Des. 8, 1628-1634.

García-Ruiz, J. M., Novella, M. L., Moreno, R. \& Gavira, J. A. (1994). J. Cryst. Growth, 232, 165-172.

Hansen, C. L., Classen, S., Berger, J. M. \& Quake, S. R. (2006). J. Am. Chem. Soc. 128, 3142-3143.

Hansen, C. L., Skordalakes, E., Berger, J. M. \& Quake, S. R. (2002). Proc. Natl Acad. Sci. USA, 99, 16531-16536.

Heijna, M. C. R., van Wamel, F. F. M., van Enckevort, W. J. P. \& Vlieg, E. (2007). Cryst. Growth Des. 7, 1999-2008.

Helliwell, J. R. \& Chayen, N. E. (2007). Nature (London), 448, 658-659. Kuroda, T., Irisawa, T. \& Ookawa, A. (1977). J. Cryst. Growth, 42, 41-46. Leng, J. \& Salmon, J. B. (2009). Lab Chip, 9, 24-34.

Lounaci, M., Rigolet, P. M., Abrahama, C., Berrea, L. \& Chen, Y. (2007). Microelectron. Eng. 84, 1758-1761.

Lounaci, M., Rigolet, P., Casquillas, G. V., Huang, H. W. \& Chen, Y. (2006). Microelectron. Eng. 83, 1673-1676.

McPherson, A. (1999). Crystallization of Biological Macromolecules, p. 437. Cold Spring Harbor Laboratory Press.

Nanev, C. N. \& Penkova, A. N. (2002). J. Cryst. Growth, 237-239, 283-288.

Sauter, C., Dhouib, K. \& Lorber, B. (2007). Cryst. Growth Des. 7, 2247-2250.

Sazaki, G., Yoshida, E., Komatsu, H., Nakada, T., Miyashita, S. \& Watanabe, K. (1997). J. Cryst. Growth, 173, 231-234.

Shim, J. U., Cristobal, G., Link, D. R., Thorsen, T. \& Fraden, S. (2007). Cryst. Growth Des. 7, 2192-2194.

Shim, J. U., Cristobal, G., Link, D. R., Thorsen, T., Jia, Y., Piattelli, K. \& Fraden, S. (2007). J. Am. Chem. Soc. 129, 8825-8835.

Talreja, S., Kim, D. Y., Mirarefi, A. Y., Zukoski, C. F. \& Kenis, P. J. A. (2005). J. Appl. Cryst. 38, 988-995.

Yadav, M. K., Gerdts, C. J., Sanishvili, R., Smith, W. W., Roach, L. S., Ismagilov, R. F., Kuhn, P. \& Stevens, R. C. (2005). J. Appl. Cryst. 38, 900-905.

Yin, D. C., Lu, H. M., Geng, L. Q., Shi, Z. H., Luo, H. M., Li, H. S., Ye, Y. J., Guo, W. H., Shang, P. \& Wakayama, N. I. (2008). J. Cryst. Growth, 310, 1206-1212.

Yin, D. C., Wakayama, N. I., Harata, K., Fujiwara, M., Kiyoshi, T., Wada, H., Niimura, N., Arai, S., Huang, W. D. \& Tanimoto, Y. (2004). J. Cryst. Growth, 270, 184-191.

Yu, Y., Liu, Y. H., Wang, X., Oberthür, D., Dierks, K. \& Betzel, C. (2010). Cryst. Res. Technol. 45, 380-386.

Zheng, B., Gerdts, C. J. \& Ismagilov, R. F. (2005). Curr. Opin. Struct. Biol. 15, 548-555.

Zheng, B., Roach, L. S. \& Ismagilov, R. F. (2003). J. Am. Chem. Soc. 125, 11170-11171.

Zheng, B., Tice, J. D. \& Ismagilov, R. F. (2004). Adv. Mater. 16, 1365-1368.

Zheng, B., Tice, J. D., Roach, L. S. \& Ismagilov, R. F. (2004). Angew. Chem. Int. Ed. 43, 2508-2511. 